Pacific

Journal of

Mathematics

HARMONIC FUNCTIONS ON MANIFOLDS WITH NONNEGATIVE RICCI CURVATURE AND LINEAR VOLUME GROWTH

Christina Sormani 


\title{
HARMONIC FUNCTIONS ON MANIFOLDS WITH NONNEGATIVE RICCI CURVATURE AND LINEAR VOLUME GROWTH
}

\author{
Christina Sormani
}

In this paper we prove that if a complete noncompact manifold with nonnegative Ricci curvature and linear volume growth has a nonconstant harmonic function of at most polynomial growth, then the manifold splits isometrically.

Lower bounds on Ricci curvature limit the volumes of sets and the existence of harmonic functions on Riemannian manifolds. In 1975, Shing Tung Yau proved that a complete noncompact manifold with nonnegative Ricci curvature has no nonconstant harmonic functions of sublinear growth [Yau2]. That is, if

$$
\limsup _{R \rightarrow \infty} \frac{\max _{B_{p}(R)}|f|}{R}=0
$$

and if $f$ is harmonic, then $f$ is a constant. In the same paper, Yau used this result to prove that a complete noncompact manifold with nonnegative Ricci curvature has at least linear volume growth,

$$
\liminf _{R \rightarrow \infty} \frac{\operatorname{Vol}\left(B_{p}(R)\right)}{R}=C \in(0, \infty] .
$$

There are many manifolds with nonnegative Ricci curvature that actually have linear volume growth

$$
\limsup _{R \rightarrow \infty} \frac{\operatorname{Vol}\left(B_{p}(R)\right)}{R}=V_{0}<\infty .
$$

Some interesting examples of such manifolds can be found in [So1].

In this paper, we prove the following theorem concerning harmonic functions on these manifolds.

Theorem 1. Let $M^{n}$ be a complete noncompact manifold with nonnegative Ricci curvature and at most linear volume growth,

$$
\limsup _{R \rightarrow \infty} \frac{\operatorname{Vol}\left(B_{p}(R)\right)}{R}=V_{0}<\infty .
$$


If there exists a nonconstant harmonic function, $f$, of polynomial growth of any given degree $q$,

$$
\Delta f=0 \quad \text { and } \quad|f(x)| \leq C\left(d(x, p)^{q}+1\right),
$$

then the manifold splits isometrically, $M^{n}=N^{n-1} \times \mathbb{R}$.

Harmonic functions of polynomial growth have been an object of study for some time. Until recently it was not known whether the space of harmonic functions of polynomial growth of a given degree on a manifold with nonnegative Ricci curvature was finite dimensional. Atsushi Kasue proved this result with various additional assumptions in [Kas1, Kas2]. Tobias Colding and Bill Minnicozzi have recently proven that this space is indeed finite dimensional with no additional assumptions [CoMin]. With our stronger condition of linear volume growth, we are able to prove that this space is only one dimensional directly using a gradient estimate of Cheng and Yau [ChgYau] and results from [So1, So2].

For background material consult the textbooks $[\mathbf{S c h Y a u}]$ and $[\mathbf{L i}]$.

The author would like to thank Professor Shing-Tung Yau for conjecturing this theorem and for his encouragement during her year at Harvard University. She would also thank to thank Professors Tobias Colding, Jeff Cheeger, William Minicozzi and Gang Tian for their continued interest in her work.

\section{Background.}

A ray, $\gamma:[0, \infty) \mapsto M^{n}$, is a geodesic which is minimal on any subsegment, $d(\gamma(t), \gamma(s))=|t-s|$. Every complete noncompact Riemannian manifold contains a ray. Given a ray, one can define its associated Busemann function, $b: M^{n} \rightarrow \mathbb{R}$, as follows:

$$
b(x)=\lim _{R \rightarrow \infty}(R-d(x, \gamma(R))) .
$$

The Busemann function is a Lipschitz function whose gradient has unit length almost everywhere, $[\mathbf{B u}]$.

In Euclidean space, the level sets of the Busemann function associated with a given ray are the planes perpendicular to the given ray. In contrast, the Busemann function defined on a manifold with nonnegative Ricci curvature and linear volume growth has compact level sets with bounded diameter growth [So1, Thm. 15]. In that paper, the author also proved that if such a manifold is not an isometrically split manifold, then the Busemann function is bounded below and $b^{-1}((-\infty, r])$ is a compact set for all $r$ [So1, Cor. 19].

Lemma 2. Let $M^{n}$ be a complete manifold with nonnegative Ricci curvature. Suppose that there is a Busemann function, $b$, which is bounded below 
by $b_{\text {min }}$ and that diameter of the level sets grows at most linearly,

$$
\operatorname{diam}\left(b^{-1}\left(b_{\min }+r\right)\right) \leq C_{D}(r+1) .
$$

Then there exists a universal constant, $C_{n}$, depending only on the dimension, $n$, such that any harmonic function, $f$, satisfies the following gradient estimate:

$$
\sup _{b^{-1}\left(\left[b_{\min }, b_{\min }+r\right)\right)}|\nabla f| \leq \frac{C_{n}}{2(r+D)} \sup _{b^{-1}\left(b_{\min }+2(r+D)\right)}|f|
$$

for all $D \geq C_{D}(r+1)$.

Proof. First note that the boundary of the compact set, $b^{-1}\left(\left[b_{\min }, r\right)\right)$, is just $b^{-1}(r)$. So, by the maximum principal, we know that for any harmonic function, $f$,

$$
\max _{b^{-1}\left(\left[b_{\min }, r\right)\right)} f \leq \max _{b^{-1}(r)} f \text { and } \min _{b^{-1}\left(\left[b_{\min }, r\right)\right)} f \geq \min _{b^{-1}(r)} f .
$$

Furthermore, Cheng and Yau have proven the following gradient estimate for harmonic functions on balls in manifolds with nonnegative Ricci curvature,

$$
\sup _{B_{p}(a / 2)}|\nabla f| \leq \frac{C_{n}}{a} \sup _{B_{p}(a)}|f|
$$

where $C_{n}$ is a universal constant depending only on the dimension, $n$. [ChgYau, Thm. 6], see also [ChgYau, p. 21, Cor. 2.2]. This will be the constant in (8). Thus, we need only relate balls to regions defined by the Busemann function to prove the theorem.

Let $x_{0}$ be a point in $b^{-1}\left(b_{\min }\right)$. Note that

$$
B_{x_{0}}(a) \subset b^{-1}\left(\left[b_{\min }, b_{\min }+a\right)\right)
$$

because the triangle inequality implies that

$$
\begin{aligned}
b(x) & =\lim _{R \rightarrow \infty} R-d(x, \gamma(R)) \\
& \leq \lim _{R \rightarrow \infty} R-d\left(x_{0}, \gamma(R)\right)+d\left(x_{0}, x\right) \\
& =b\left(x_{0}\right)+d\left(x_{0}, x\right) .
\end{aligned}
$$

On the other hand, using our diameter bound in (7), we claim that

$$
b^{-1}\left(\left[b_{\min }, b_{\min }+r\right)\right) \subset B_{x_{0}}(r+D) \quad \forall D \geq C_{D}(r+1) .
$$

To see this we will construct a ray, $\sigma$, emanating from $x_{0}$ such that for all $t \geq 0, \sigma(t) \in b^{-1}\left(r_{\min }+t\right)$. Then, for any $y \in b^{-1}\left(\left[b_{\min }, b_{\min }+r\right)\right)$, we let $t=b(y)$ and we have

$$
d\left(x_{0}, y\right) \leq d\left(x_{0}, \sigma(t)\right)+d(\sigma(t), y) \leq t+\operatorname{diam}\left(b^{-1}\left(r_{\min }+t\right)\right) \leq r+D
$$


which implies (12). The ray, $\sigma$, is constructed by taking a limit of minimal geodesics, $\sigma_{i}$, from $x_{0}$ to $\gamma\left(R_{i}\right)$. A subsequence of such a sequence of minimal geodesics always converges. The limit ray satisfies the required property,

$$
\begin{aligned}
b(\sigma(t)) & =\lim _{i \rightarrow \infty} b\left(\sigma_{i}(t)\right)=\lim _{i \rightarrow \infty} \lim _{R \rightarrow \infty} R-d\left(\sigma_{i}(t), \gamma(R)\right) \\
& =\lim _{R \rightarrow \infty} R-\left(d\left(\sigma_{i}(0), \gamma(R)\right)-t\right)=b\left(x_{0}\right)+t .
\end{aligned}
$$

We can now combine the relationships between Busemann regions and balls, (12) and (11), with the gradient estimate, (10), and the maximum principal, (9), to prove the lemma. That is, for all $D \geq C_{D}(r+1)$, we have

$$
\begin{aligned}
\sup _{b^{-1}\left(\left[b_{\min }, b_{\min }+r\right)\right)}|\nabla f| & \leq \sup _{B_{x_{0}}(r+D)}|\nabla f| \text { by }(12), \\
& \leq \frac{C_{n}}{2(r+D)} \sup _{B_{x_{0}}(2(r+D))}|f| \\
& \leq \frac{C_{n}}{2(r+D)} \sup _{b^{-1}\left(\left[b_{\min }, b_{\min }+2(r+D)\right)\right)}|f| \\
& \leq \frac{C_{n}}{2(r+D)} \sup _{b^{-1}\left(b_{\min }+2(r+D)\right)}|f| .
\end{aligned}
$$

We employ this lemma and elements of the proof to prove our theorem.

\section{Proof of the Theorem.}

The given manifold, $M^{n}$, has nonnegative Ricci curvature and linear volume growth. We will assume that $M^{n}$ doesn't split isometrically and demonstrate that the harmonic functions of polynomial growth must be constant. Since the manifold doesn't split isometrically and has linear volume growth, any Busemann function, $b$, has a minimum value by [So1, Cor. 23]. Furthermore, by [So2, Thm. 1], the diameters of the level sets of the Busemann function grow sublinearly. Thus we satisfy the hypothesis of Lemma 2 with $C_{D}=1$ in $(7)$.

Let $M(r)=\max _{b^{-1}\left(b_{\min }+r\right)}|f|$, where $f$ is a harmonic function of polynomial growth. Note that $M$ is an nondecreasing function by the maximum principal, (9). By the lemma, we know that for all $r \geq b_{\min }$ and for all $D \geq(r+1)$, we can bound the gradient of $f$ in terms of $M$,

$$
\sup _{b^{-1}\left(\left[b_{\min }, b_{\min }+r\right)\right)}|\nabla f| \leq \frac{C_{n} M(2(r+D))}{2(r+D)} .
$$

Since $b^{-1}(r)$ is compact, there exists $x_{r}, y_{r} \in b^{-1}\left(b_{\min }+r\right)$ such that

$$
f\left(x_{r}\right)=\min _{b^{-1}\left(b_{\min }+r\right)} f \quad \text { and } \quad f\left(y_{r}\right)=\max _{b^{-1}\left(b_{\min }+r\right)} f .
$$


We claim that, for $r$ sufficiently large, $M(r) \leq f\left(y_{r}\right)-f\left(x_{r}\right)$.

First recall that if $f$ is a positive or negative harmonic function on a manifold with nonnegative Ricci curvature, then $f$ must be constant [Yau1, Cor. 1]. So there exists a point $z \in M^{n}$ such that $f(z)=0$. Thus, by the maximum principal, if $r \geq b(z)$ we know that $f\left(y_{r}\right) \geq 0$ and $f\left(x_{r}\right) \leq 0$. So $M(r)=\max \left(f\left(y_{r}\right),-f\left(x_{r}\right)\right) \leq f\left(y_{r}\right)-f\left(x_{r}\right)$.

We can now estimate $M(r)$ from above in terms of the gradient of $f$ and the diameter of the level set, $b^{-1}(r)$. First we join $x_{r}$ to $y_{r}$ by a smooth minimal geodesic, $\gamma_{r}$. Note that the length of $\gamma_{r}$, is less than or equal to diam $\left(b^{-1}(r)\right)$ by the definition of diameter. So $\gamma_{r} \subset b^{-1}\left(b_{\min }, r+\right.$ diam $\left.\left(b^{-1}(r)\right)\right)$. Thus for all $r \geq b(z)$, for all $D \geq(r+1)$, we have

$$
\begin{aligned}
& M(r) \leq f\left(y_{r}\right)-f\left(x_{r}\right) \\
& \leq \int_{0}^{L\left(\gamma_{r}\right)} \frac{d}{d t} f(\gamma(t)) d t \\
& \leq \int_{0}^{L\left(\gamma_{r}\right)}|\nabla f|\left|\gamma^{\prime}(t)\right| d t \\
& \leq \sup _{b^{-1}\left(\left[b_{m i n}, r+\operatorname{diam}\left(b^{-1}(r)\right)\right)\right)}|\nabla f| \int_{0}^{L\left(\gamma_{r}\right)}\left|\gamma^{\prime}(t)\right| d t \\
& \leq \frac{C_{n} M\left(2\left(r+\operatorname{diam}\left(b^{-1}(r)\right)+D\right)\right)}{2\left(r+\operatorname{diam}\left(b^{-1}(r)\right)+D\right)} \operatorname{diam}\left(b^{-1}(r)\right) \\
& \leq C_{n} M\left(2\left(r+\operatorname{diam}\left(b^{-1}(r)\right)+D\right)\right) \frac{\operatorname{diam}\left(b^{-1}(r)\right)}{2 r} \\
& \leq C_{n} M(2(r+(r+1)+D)) \frac{\operatorname{diam}\left(b^{-1}(r)\right)}{2 r} .
\end{aligned}
$$

Setting $D=r+1$ and taking $r \geq 1$, we have

$$
M(r) \leq C_{n} M(6 r) \frac{\operatorname{diam}\left(b^{-1}(r)\right)}{2 r} .
$$

Recall that our manifold has sublinear diameter growth by [So2, Thm. 1]. So, given any $\delta>0$, we can find $R_{\delta} \geq 1$ such that

$$
\frac{\operatorname{diam}\left(b^{-1}(r)\right)}{2 r}<\delta \quad \forall r \geq R_{\delta} .
$$

Then, for all $k \in \mathbf{N}$ and for all $R \geq R_{\delta}$, we have

$$
M(R) \leq C_{n} M(6 R) \delta \leq \cdots \leq C_{n}^{k} M\left(6^{k} R\right) \delta^{k} .
$$

Now $f$ has polynomial growth of order $q$, (5), so

$$
M(r)=\max _{x \in b^{-1}\left(b_{\min }+r\right)}|f(x)| \leq \max _{x \in b^{-1}\left(b_{\min }+r\right)} C\left(d\left(x, x_{0}\right)^{q}+1\right) .
$$


Applying (12) with $C_{D}=1$ and $D=C_{D}(r+1)$, we get

$$
M(r) \leq C\left((r+(r+1))^{q}+1\right) \leq C\left(6 r^{2}\right)^{q} \quad \forall r \geq 1 .
$$

Substituting this information into (17), we get

$$
\begin{aligned}
M(R) & \leq C_{n}^{k} C\left(6\left(6^{k} R\right)^{2}\right)^{q} \delta^{k} \\
& \leq C 6^{q} R^{2 q}\left(C_{n} 6^{2 q} \delta\right)^{k} \quad \forall R \geq R_{\delta} .
\end{aligned}
$$

Fix $\delta=1 /\left(2 C_{n} 6^{2 q}\right)$, so $R_{\delta}$ is fixed by (16). Then, for all $R \geq R_{\delta}$, we have

$$
M(R) \leq \lim _{k \rightarrow \infty} C 6^{q} R^{2 q}(1 / 2)^{k}=0 .
$$

Since $M(r)$ is nondecreasing and nonnegative, $M(r)=0$ everywhere. Thus, $f$ is a constant.

\section{References}

[Bu] H. Busemann, The Geometry of Geodesics, Academic Press Inc., New York, N. Y., 1955.

[ChGl] J. Cheeger and D. Gromoll, The splitting theorem for manifolds of nonnegative Ricci curvature, J. Differential Geometry, 6 (1971/72), 119-128.

[ChgYau] S.Y. Cheng and S.T. Yau, Differential equations on Riemannian manifolds and their geometric applications, Communications on Pure and Applied Mathematics, Vol. XXVII, (1975), 333-354.

[CoMin] T. Colding and W. Minicozzi, Harmonic functions on manifolds, Ann. of Math., 146(3) (1997), 725-747.

[Kas1] A. Kasue, Harmonic functions of polynomial growth on complete Riemannian manifolds I, Proc. Symp. Pure Math., Vol. 54, part II, (1993), 281-290.

[Kas2] Harmonic functions of polynomial growth on complete Riemannian manifolds II, J. Math. Soc. Japan, Vol. 47, (1995), 37-65.

[Li] P. Li, Lecture Notes on Geometric Analysis, Lecture Notes Series, 6, Seoul National University, Research Institute of Mathematics, Global Analysis Research Center, Seoul, 1993.

[SchYau] R. Schoen and S.-T. Yau, Lectures on Differential Geometry, Conference Proceedings and Lecture Notes in Geometry and Topology, Vol. 1, International Press Inc., Cambridge, MA, 1994.

[So1] C. Sormani, Busemann functions on manifolds with lower Ricci curvature bounds and minimal volume growth, Journal of Differential Geometry, 48 (1998), 557-585.

[So2] - The rigidity and almost rigidity of manifolds with minimal volume growth, Communications in Analysis and Geometry, to appear.

[So3] _ Complete Noncompact Manifolds with Lower Bounds on Ricci Curvature and Minimal Volume Growth, PhD Thesis, CIMS, 1996. 
[Yau1] S.T. Yau. Harmonic functions on complete Riemannian manifolds, Comm. Pure Appl. Math., 28 (1975), 201-228.

[Yau2] Some function theoretic properties of complete Riemannian manifold and their applications to geometry, Indiana University Mathematics Journal, 25(7) (1976), 659-670.

Received April 3, 1998.

JoHns Hopkins UnIVERSITY

BALTimore, MD 21218

E-mail address: sormanic@member.ams.org 